\title{
Effects of nonprotein amino acids on survival and locomotion of Osmia bicornis
}

\begin{abstract}
A. Felicioli*, S. Sagona*, M. Galloni†, L. Bortolottił, G. Bogo†‡, M. Guarnieri§ and M. Nepi (D)

${ }^{*}$ Department of Veterinary Science, University of Pisa, Pisa, Italy; †Department of Biological, Geological and Environmental Sciences, University of Bologna, Bologna, Italy; ¥Council for Agricultural Research and Economics, Research Centre for Agriculture and Environment (CREA$A A)$, Bologna, Italy; and §Department of Life Sciences, University of Siena, Siena, Italy
\end{abstract}

\begin{abstract}
To investigate the effects of two nectar nonprotein amino acids, $b$-alanine and $g$-aminobutyric acid (GABA), on Osmia bicornis survival and locomotion, two groups of caged bees were fed with sugar syrup enriched with $b$-alanine and GABA, respectively. A further control group was fed with sugar syrup. Five behavioural categories were chosen according to the principle of parsimony and intrinsic unitary consistency from start to end, and recorded by scan sampling: two states (remaining under paper or in tubes) and three events (walking on net, feeding from flower and flying). We also analysed the amino acid content of haemolymph sampled from an additional 45 bees fed the same diets (15 per diet type). Bees fed with $b$-alanine had a significantly shorter survival time than those fed with the control and GABA diets. The GABA diet induced higher levels of locomotion than $b$-alanine. The former nonprotein amino acid was only detected in the haemolymph of bees fed GABA. The results suggest that insects consuming nonprotein amino-acid-rich diets absorb and transfer these substances to the haemolymph and that nonprotein amino acids affect survival and locomotion. Ecological consequences are discussed in the framework ofplant reproductive biology.
\end{abstract}

Correspondence: Massimo Nepi, Department of Life Sciences, University of Siena, Via Pier Andrea Mattioli 4, Siena 53100, Italy. Tel.: 1390577233767; fax: 1390577232860; e-mail: massimo.nepi@unisi.it
Keywords: GABA, b-alanine, nonprotein amino acids, Osmia bicornis, survival, locomotion.

\section{Introduction}

Floral nectar is the most commonly used fuel for the high energy demands of insect flight (Nicolson \& Thorburg, 2007). Up to $70-80 \%$ (weight/weight) of floral nectar consists of simple carbohydrates, mostly sucrose, glucose and fructose (Nicolson \& Thorburg, 2007). Amino acids are generally much less abundant than sugars, ranging from 1/100-1/1000 of nectar sugar concentrations (Heil, 2011; Nepi et al., 2012); however, they contribute to the nutritional importance of nectar as all the essential amino acids have been found in this secretion (reviewed by Nicolson, 2007). Amino acids also contribute to nectar taste, stimulating the labellar chemoreceptors of insects (Nicolson, 2007). Specific preferences for single or for a mixture of amino acids have been reported for some insects (González-Teuber \& Heil, 2009; Bertazzini et al., 2010). Although their concentrations are much less than those of sugars, nectar amino acids may have physiological effects in insects. For example, it has been reported that non-essential amino acids in nectar enhance butterfly fecundity and appear to compensate butterflies after the poor food of the larval phase (Mevi-Schttz \& Erhardt, 2005). Amino acids may also affect insect longevity, but the effect may be positive or negative. For example, females of the mosquito Culex quinquefasciatus fed diets containing nectar carbohydrates and amino acids lived significantly longer than females fed diets only containing sugar (Vrzal et al., 2010). By contrast, honeybee survival is negatively affected by diets rich in essential amino acids, although the effect depends on the bee's age (Paoli et al., 2014). Other nutrients, such as lipids, vitamins and minerals, may also affect bee health at individual and colony levels (Haydak, 1970).

All these functions of nectar amino acids and most of the many studies analysing nectar amino acids concern protein amino acids, i.e. amino acids that can be used for protein synthesis. A few nectar chemistry studies 
simply report the presence of certain nonprotein amino acids (NPAAs, i.e. amino acids that are not constituents of proteins) without expressing any hypothesis about their ecological role or effect on flower visitors. However, NPAAs can be very abundant (Kaczorowski et al., 2005; Rossi et al., 2014), sometimes accounting for $30-50 \%$ of the total amino acids found in nectar (Nepi et al., 2012; Nocentini et al., 2012).

Gamma-aminobutyric acid (GABA) and $b$-alanine are the nonprotein amino acids most frequently reported in nectar (Nepi, 2014). It was recently postulated that nectar NPAAs, and particularly GABA and $b$-alanine, could be of ecological importance, affecting the foraging behaviour of pollinators through effects on the insect nervous system, regulating feeding rate and promoting muscle activity (Nepi, 2014). GABA and b-alanine are important neurotransmitters in insect nervous systems (Nepi, 2014 and references therein). Insects have an open body cavity, and all their organs and tissues, including the brain, are immersed in haemolymph. Thus brain and nervous system activity depend on the metabolites in haemolymph (Lu et al., 2014): these metabolites vary in relation to diet and to developmental and physiological state (Gillot, 2005). In invertebrates, GABAreceptors are located peripherally in muscle and neuromuscular junctions, where they are bathed in haemolymph (Bown et al., 2006). Thus, insects are presumably sensitive to changes in NPAAs having neural and muscular activity whose concentration in haemolymph may increase with consumption of nectar. These new hypotheses prompted us to study the effect of diets enriched with the two major nectar NPAAs, i.e. GABA and $b$ alanine, on Osmia bicornis, a univoltine bee. Its imago flying season extends from February to April. The imago only feeds on nectar and collects pollen to provision the nest with food for the larvae once they hatch (Tasei \& Picart, 1973; Krunic et al., 2005). Owing to its solitary life cycle and tolerance to caging, O. bicornis is a suitable species for observing and measuring behaviours. Our study aimed at acquiring new information on: (1) the effects of an NPAA-enriched diet on survival and locomotion of $O$. bicornis and (2) the presence of dietary NPAAs in haemolymph.

\section{Results}

\section{Survival and behavioural observations}

The maximum survival times of bees in the $b$-alanine, GABA and control groups were 42, 52 and 63 days, respectively (Fig. 1). A significant difference was only found between the control and $b$-alanine groups; no significant difference was found between the GABA and $b$ alanine groups or the control and GABA groups (Fig. 1).

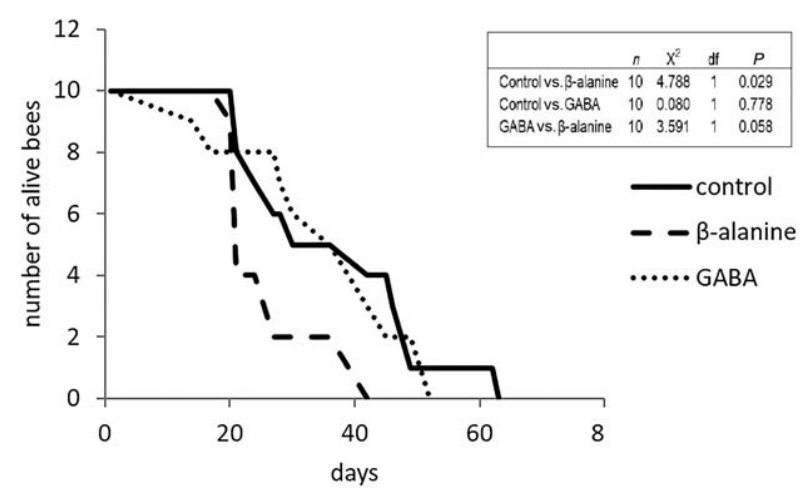

Figure 1. Survival curves of the three experimental groups of Osmia bicornis. The $b$-alanine-enriched diet was associated with shorter survival times. Values of $\mathrm{v}^{2}$ and $P$ are reported in the inset. GABA, $g$-aminobutyric acid.

The behavioural parameters were significantly affected by diet (Fig. 2). The number of bees 'walking on net' was significantly higher in the GABA than in the $b$ alanine group, and neither was significantly different from the control (Fig. 2A). No significant difference was found between treatment groups for 'flying' (Fig. 2B). The only significant difference for 'visiting flower' was between the control and GABA groups (Fig. 2C). Amongst the state parameters, the number of bees 'remaining under paper' was not significantly different between groups (Fig. 2D). The number of bees 'remaining in tube' was fewer in the GABA and $b$-alanine groups than in the control group (Fig. 2E).

\section{Haemolymph amino acids}

The most abundant protein amino acids in the haemolymph of newly emerged 0 . bicornis (time 0 ) were the non-essential proline and alanine (53 and $7 \%$, respectively), and the essential arginine and histidine (22 and $4 \%$, respectively) (Table 1 ). All other protein amino acids were generally less than $2 \%$. Amongst the NPAAs considered, we only found $b$-alanine $(2.4 \%)$. Both diets induced detectable variations in the amino acid composition of haemolymph. Those of arginine and proline were the most evident. Proline showed a decreasing trend in both diet groups and arginine an increasing trend (Fig. $3 A-C)$. The relative percentage of $b$-alanine increased in both diet groups, more sharply in that supplemented with $b$-alanine (Fig. 3D-F). GABA was only detectable (up to $5.06 \%$ ) after $24 \mathrm{~h}$ in bees fed with the GABAenriched diet and decreased to 0 at $96 \mathrm{~h}$ (Fig. 3F).

\section{Discussion}

\section{Diet and insect survival}

The high concentration of GABA in the feeding solution did not affect the lifespan of newly emerged female 

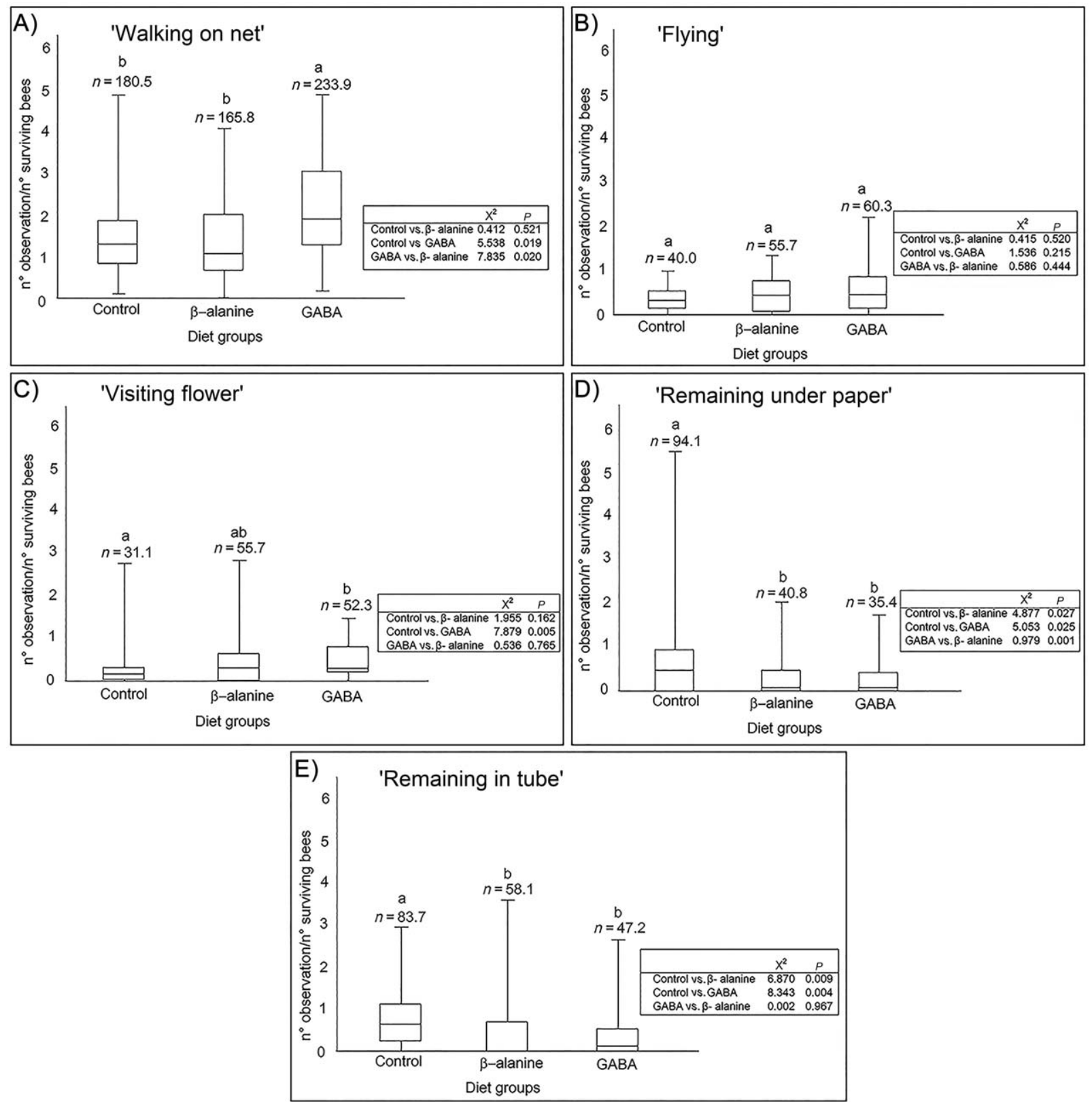

Figure 2. Box-plot diagrams of behavioural data in relation to the three experimental diets. (A) Event 'walking on net': the number of bees performing this behaviour was significantly higher in the $g$-aminobutyric acid (GABA) than in the $b$-alanine diet group. (B) Event 'flying': there were no significant differences between groups. (C) Event 'visiting flower': the number of bees performing this behaviour was high in the GABA group with respect to the control. (D) State 'remaining under paper': no significant differences were recorded between groups. (E) Event 'remaining in tube': the number of bees performing this behaviour was low in GABA and $b$-alanine groups with respect to the control. Values of the Kruskal-Wallis test and $P$-values from Bonferroni-corrected Mann-Whitney pairwise comparison are reported in the inset of each diagram. $n$ over bars indicates the total number of recordings of each behavioural parameter for all observation sessions (33 days for each diet). Different letters indicate significant differences between diet groups $(P<0.05)$.

imagoes of O. bicornis, whereas lifespan decreased in those fed $b$-alanine.

To the best of our knowledge, no similar studies have been reported for this species or genus. The only other study reporting the effect of consumption of a NPAA was performed on a very different insect, the oblique-banded leafroller larva (Bown et al., 2006). These caterpillars show a significant reduction in survival if fed with 11.6 mM GABA (Bown et al., 2006). We used a similar concentration of GABA (15 mM) and although there was a slight reduction in bee survival, it was not significantly different from the control (52 vs. 62 days).

Dietary enrichment with essential protein amino acids instead of NPAAs was tested on another bee species 
A)

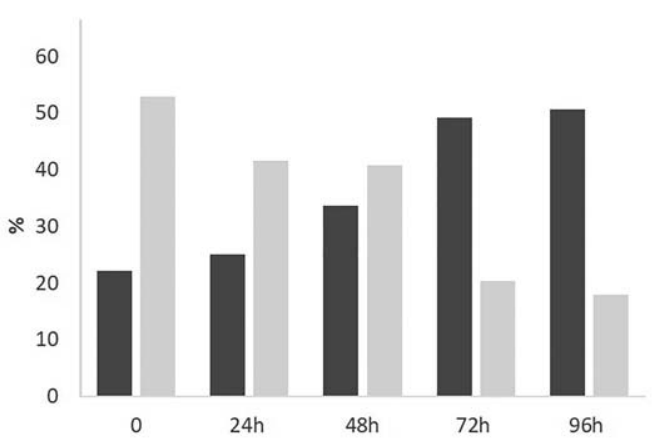

B)

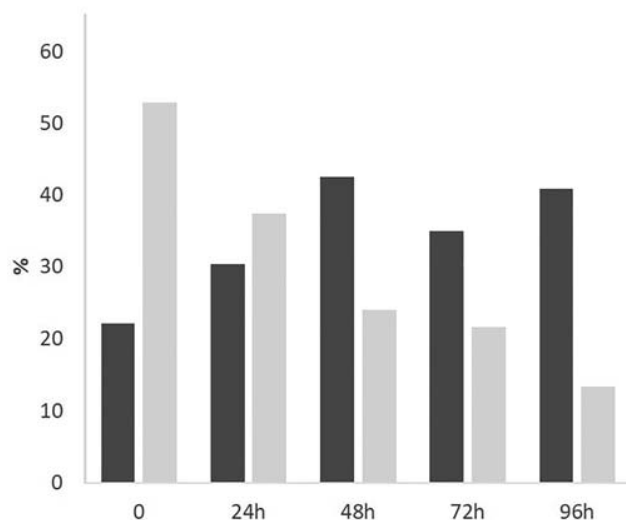

C)

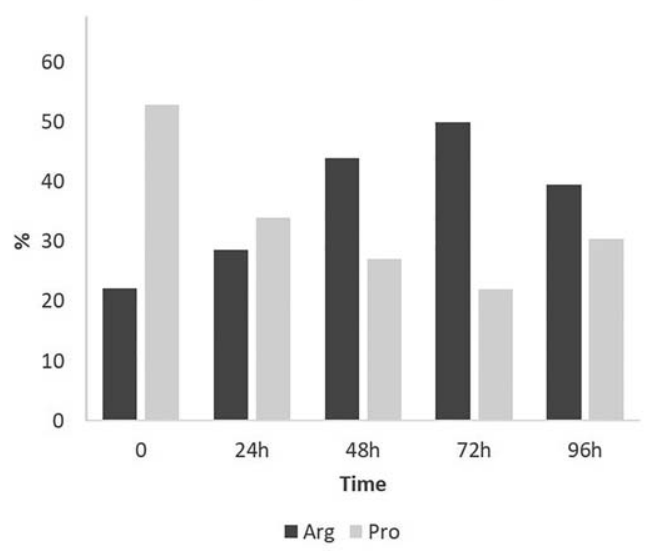

D)

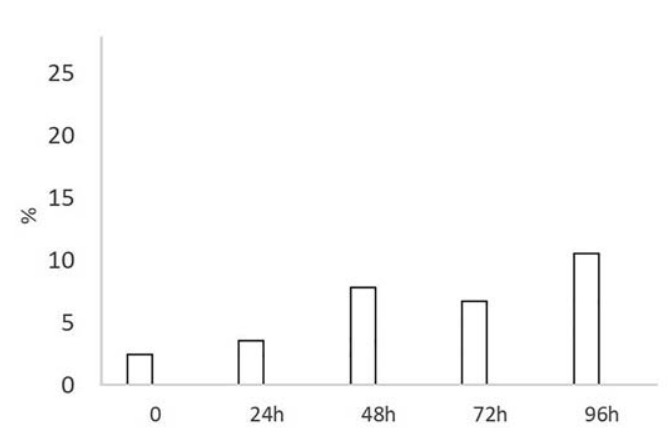

E)

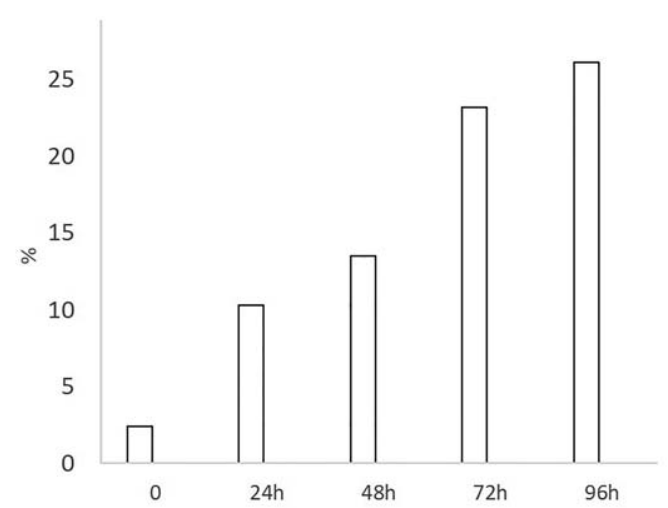

F)

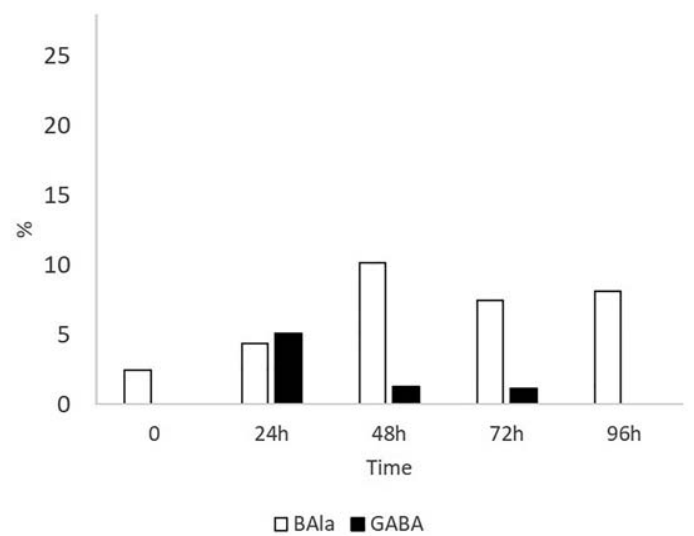

Figure 3. Amino acid percentages over time in haemolymph of Osmia bicornis females bees fed experimental diets. (A-C) arginine and proline in control, $b$-alanine (BAla) and $g$-aminobutyric acid (GABA) groups, respectively. (D-F) $b$-alanine and GABA in control, $b$-alanine and GABA groups, respectively. Under our experimental conditions, GABA was only detected in haemolymph of bees fed the GABA-enriched diet.

(Apis mellifera) by Paoli et al. (2014). A significant reduction in longevity associated with the essential amino acid-rich diet was observed in young as well as forager honeybees.

\section{Diet and insect behaviour}

Bees fed the GABA-enriched diet showed higher motor activity than controls. As we did not measure food consumption, there are two main hypotheses for this result.
First, GABA could increase the attraction of bees. Consequently, bees may consume more sugar-based syrup and therefore show greater motor activity. GABA is reported to stimulate feeding in some herbivorous insects. In these insects GABA stimulates taste chemoreceptors sensitive to sugars, and this results in increased feeding behaviour (Shoonhoven et al., 2005). These receptors appeared to be sensitive to a GABA concentration (0.01 M, Mitchell \& Harrison, 1984) similar to that used in our experiments $(0.015 \mathrm{M})$. Second, 
Table 1. Relative percentages of amino acids in haemolymph of Osmia bicornis females fed different diets [sugar syrup, sugar syrup with b-alanine (b-ala) and sugar syrup with c-aminobutyric acid (GABA)].

\begin{tabular}{|c|c|c|c|c|c|c|c|c|c|c|c|c|c|}
\hline Amino acid & $\begin{array}{c}\text { Control } \\
0\end{array}$ & $\begin{array}{c}\text { Control } \\
24\end{array}$ & $\begin{array}{c}\text { Control } \\
48\end{array}$ & $\begin{array}{c}\text { Control } \\
72\end{array}$ & $\begin{array}{c}\text { Control } \\
96\end{array}$ & $\begin{array}{c}\text { GABA } \\
24\end{array}$ & $\begin{array}{c}\text { GABA } \\
48\end{array}$ & $\begin{array}{c}\text { GABA } \\
72\end{array}$ & $\begin{array}{c}\text { GABA } \\
96\end{array}$ & $\begin{array}{c}\text { b-ala } \\
24\end{array}$ & $\begin{array}{c}\text { b-ala } \\
48\end{array}$ & $\begin{array}{c}\text { b-ala } \\
72\end{array}$ & $\begin{array}{c}\text { b-ala } \\
96\end{array}$ \\
\hline Ser & 0.87 & 0.67 & 0.36 & 0.63 & 0.78 & 0.63 & 0.61 & 0.38 & 0.39 & 0.48 & 0.43 & 0.34 & 0.26 \\
\hline Glu & 0.68 & 1.34 & 0.68 & 2.85 & 1.56 & 0.86 & 1.70 & 2.09 & 1.07 & 1.21 & 1.12 & 0.83 & 0.59 \\
\hline Gly & 0.97 & 0.96 & 1.01 & 0.88 & 1.84 & 0.95 & 0.68 & 0.93 & 0.84 & 1.11 & 0.87 & 0.58 & 0.93 \\
\hline $\mathrm{His}$ & 4.14 & 10.24 & 4.77 & 8.80 & 9.60 & 8.05 & 6.33 & 6.92 & 10.30 & 7.43 & 8.34 & 11.14 & 12.07 \\
\hline Arg & 22.18 & 25.02 & 33.55 & 49.26 & 50.62 & 28.52 & 43.98 & 49.98 & 39.45 & 30.35 & 42.47 & 34.98 & 40.80 \\
\hline Ala & 7.04 & 6.34 & 4.07 & 5.57 & 3.80 & 9.05 & 4.22 & 4.87 & 4.69 & 5.19 & 3.71 & 3.29 & 2.96 \\
\hline Pro & 52.87 & 41.65 & 40.68 & 20.30 & 17.91 & 33.98 & 26.99 & 22.04 & 30.39 & 37.38 & 24.01 & 21.67 & 13.34 \\
\hline Tyr & 0.12 & 1.10 & 1.80 & 1.07 & 0.81 & 1.55 & 0.99 & 1.10 & 1.07 & 0.91 & 1.28 & 1.14 & 0.67 \\
\hline Val & 2.17 & 1.71 & 1.37 & 0.52 & 0.37 & 0.95 & 0.68 & 0.58 & 0.65 & 1.13 & 0.52 & 0.63 & 0.39 \\
\hline Met & 1.83 & 2.03 & 1.50 & 1.04 & 0.84 & 1.65 & 0.92 & 0.93 & 0.90 & 1.54 & 1.28 & 1.01 & 0.42 \\
\hline Lys & 1.18 & 1.06 & 0.49 & 1.23 & 0.69 & 1.93 & 0.54 & 0.58 & 0.62 & 1.18 & 1.41 & 0.47 & 0.65 \\
\hline Ile & 0.97 & 0.73 & 0.53 & 0.27 & 0.16 & 0.56 & 0.27 & 0.27 & 0.36 & 0.45 & 0.25 & 0.22 & 0.28 \\
\hline Leu & 1.23 & 0.77 & 0.30 & 0.14 & 0.06 & 0.49 & 0.10 & 0.21 & 0.25 & 0.40 & 0.18 & 0.09 & 0.10 \\
\hline Phe & 0.92 & 0.97 & 1.09 & 0.47 & 0.44 & 1.00 & 0.51 & 0.51 & 0.59 & 0.63 & 0.62 & 0.40 & 0.29 \\
\hline b-Ala & 2.43 & 3.57 & 7.79 & 6.72 & 10.53 & 4.36 & 10.18 & 7.44 & 8.14 & 10.30 & 13.51 & 23.20 & 26.08 \\
\hline GABA & & & & & & 5.06 & 1.29 & 1.17 & 0.00 & & & & \\
\hline$\%$ Essential protein amino acids & 34.62 & 44.10 & 43.61 & 61.74 & 62.77 & 43.14 & 53.34 & 59.99 & 53.11 & 43.12 & 55.07 & 48.95 & 55.01 \\
\hline $\begin{array}{l}\% \text { Non-essential protein } \\
\text { amino acids }\end{array}$ & 62.56 & 52.04 & 48.60 & 31.29 & 26.70 & 47.02 & 35.19 & 31.40 & 38.44 & 46.27 & 31.42 & 27.86 & 18.75 \\
\hline$\%$ Nonprotein amino acids & 2.82 & 3.86 & 7.79 & 6.97 & 10.53 & 9.84 & 11.47 & 8.60 & 8.45 & 10.60 & 13.51 & 23.20 & 26.25 \\
\hline
\end{tabular}

The numbers after 'Control', 'GABA' and 'b-alanine' in the column headings refer to the times (in h) at which the haemolymph was sampled.

Essential amino acids reported by de Groot (1953) for honeybees are in bold.

GABA could stimulate the motor activity of bees through its neurotransmitter function. This is apparently in contrast with the inhibitory function of GABA in central and peripheral neurotransmission in insects (Breer \& Heilgenberg, 1985; Schnee et al., 1997; Bown et al., 2006), suggesting two hypotheses: (1) the action of GABA may be not univocal as it can switch from inhibitory to excitatory as reported in some vertebrates (Ben-Ari et al., 2012); however, it is not clear whether this excitatory role exists in insects; (2) high concentrations of GABA in haemolymph and the pure sugar diet could play a pivotal role in the GABA shunt (Olsen \& DeLorey, 1999). In this metabolic pathway, GABA is converted into glutamate via succinic acid and $a$-ketoglutarate. Glutamate has an excitatory function at honeybee neuromuscular junctions
(Gauthier \& Grfnewald, 2011). With regard to locomotion, the GABA-enriched diet affected 'walking on net' as well as 'visiting flower', whereas 'flying' was not significantly affected. Its lack of effect on flying needs to be verified using a tool specifically conceived and set up to examine this behavioural event.

\section{Diet and haemolymph composition}

Proline was the most abundant amino acid in the haemolymph of newly emerged $O$. bicornis $(53 \%)$. This finding is in line with the percentage $(50 \%)$ reported by Crailsheim and Leonhard (1997) in newly emerged worker honeybees. Proline is involved in the flight metabolism of forager honeybees (Micheu et al., 2000;

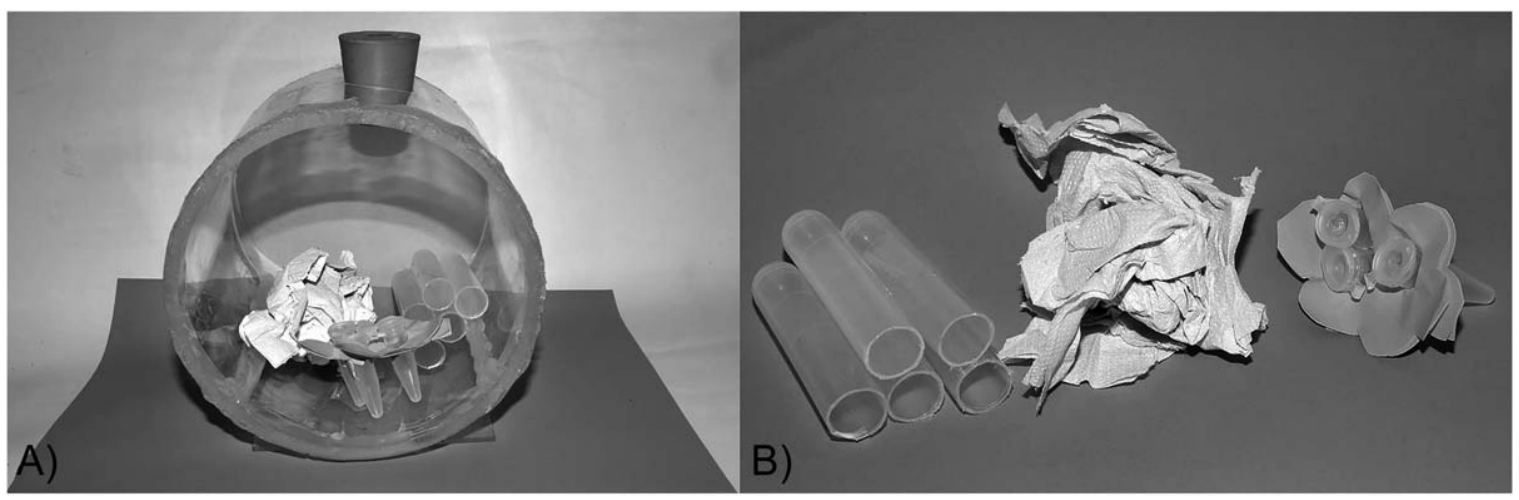

Figure 4. Details of cage: (A) rearing cage and (B) plastic tubes, paper and artificial flower connected to Eppendorf tubes containing the feeding solution. 
Carter et al., 2006) and in egg laying by the queen (Hrassnigg et al., 2003). Forager honeybees fly to collect nectar and pollen whereas the queen has the role of laying eggs. Females of $O$. bicornis perform both activities, flight and egg laying (Raw, 1972; Felicioli et al., 2002), and proline may be involved in both.

Arginine was the second most abundant amino acid found in the haemolymph, with an arginine : proline ratio of 0.41 . This value is higher than those recorded by Milani (1988) for worker and queen honeybees.

Beta-alanine has been reported to have a ratio to proline of 0.11 in honeybee haemolymph (Milani, 1988). In the haemolymph of newly emerged 0 . bicornis, the $b$ alanine : proline ratio was 0.046 , a similar order of magnitude to that of honeybees. A GABA : proline ratio of 0.06 has been reported in hive worker honeybees (Milani, 1988) but was not found in O. bicornis unless the bees were fed a GABA-enriched diet. As the results concern hive worker honeybees and caged $O$. bicornis bees fed an artificial diet, this difference needs to be accepted with caution. In the light of these considerations, further investigation into the presence of GABA in insect haemolymph is needed, considering caged worker honeybees fed an artificial diet and free-ranging $O$. bicornis bees. GABA has also been detected in the haemolymph of the cockroach Blattella germanica fed an artificial diet enriched with this NPAA (Auclair, 1959). Individuals of O. bicornis fed a GABA-enriched diet showed an initial increase in GABA levels at $24 \mathrm{~h}$, followed by a steady decrease despite the fact that the bees continued to feed on the GABA-rich solution. This suggests a homeostatic response to GABA increase in the haemolymph. Under our experimental conditions (exclusively sugar diet), the GABA shunt hypothesis is in line with this physiological response and the behavioural effect (increased 'walking on net'), as mentioned above. GABA homeostasis also plays a role in certain aspects of brain function in mammals (Wyatt, 2016). In herbivorous insect pests, such as larvae of the obliquebanded leafroller, it is reported that GABA-activated $\mathrm{Cl}^{-}$ channels bind excess GABA taken up by the haemolymph from a GABA-enriched diet (Bown et al., 2006).

To the best of our knowledge, this study is the first to test the effect of NPAAs on nectar-consuming insects. It is a further demonstration that insect diet may affect haemolymph composition. Specifically, and more interestingly, it demonstrates that dietary $b$-alanine and GABA may accumulate in the haemolymph of $O$. bicornis. The accumulation pattern was different for the two NPAAs, suggesting a sort of homeostatic regulation for GABA only. We are aware that the un-natural conditions in which the bees were kept and the use of high concentrations of NPAAs [20-fold higher than those reported in floral nectar (Nepi, 2014)] mean that the outcome of our experiments can hardly be transposed to a natural ecological context. Nonetheless, our results could be an initial basis for monitoring GABA and $b$-alanine in the haemolymph of free-ranging $O$. bicornis individuals in order to investigate the possible link between NPAAs in nectars and the foraging activity of insects. Insect foraging of flowers is responsible for pollen transport and gene flow within and between plant populations, which in turn affects their genetic structure.

\section{Experimental procedures}

\section{Survival rate and behavioural observations}

O. bicornis females were obtained from an established population in the garden of the Veterinary Science Department of Pisa University $\left(43842^{0} 24.98^{00} \mathrm{~N}, 10824046.69^{\circ} \mathrm{E}\right)$. Since 2001 , a yearly release and rearing campaign (Felicioli, 2000) has been conducted using artificial nests in a bee hotel. Release and rearing involves using cocoons with a 2:1 (male : female) sex ratio obtained from a population reared the previous year or from a nest trapping campaign. Nest trapping consists of capturing local wild O. bicornis populations by means of artificial nests. The artificial nests are of the artificial trap nest and reed nest type (Krunić et al., 2005; Felicioli et al., 2017). Three quarters of all the artificial nests are opened in November of each year. The cocoons are stripped and divided by species and sex. All cocoons of a given species are pooled and stored at $48 \mathrm{C}$ until use in February (Yin et al., 2013). Two groups of 30 and 45 cocoons, each containing a female of $O$. bicornis, were sampled randomly from the pool. The first group of 30 cocoons was divided into three subgroups of 10 bees. At the beginning of March, each subgroup was located in the field in a cylindrical plastic cage (15 cm diameter $315 \mathrm{~cm}$ long) closed by a net at both ends until emergence occurred. All the females emerged within a single day. The cage contained a feeder (a yellow cardboard flower connected to three eppendorf tubes containing the syrup, hereafter called 'flower'), plastic tubes and paper so that the bees could hide and walk (Fig. 4). The groups were fed with the following diets: sugar syrup [sucrose $20 \%$ weight/volume (w/v), control group], $46 \mathrm{mM} b$-alanine in sugar syrup ( $b$-ala group) and $15 \mathrm{mM}$ GABA in sugar syrup (GABA group). These concentrations were chosen for the following reasons:

1. $20 \% \mathrm{w} / \mathrm{v}$ sucrose for the control group because this is the concentration used successfully for rearing honeybees in cages (Bertazzini et al., 2010; Mazzei et al., 2016).

2. $46 \mathrm{mM}$ b-alanine and $15 \mathrm{mM}$ GABA because these concentrations are 20-fold those found in the field (Nepi, 2014) and increase the probability of obtaining information about repulsion/attraction, mortality/survival and behavioural effects.

The survival time of the bees in each cage was recorded. The number of bees manifesting three events (walking on net, flying, and visiting the artificial flower, hereafter 'visiting flower') and two states (remaining under paper or in tube) was counted by scan sampling ( 5 min every hour from 9 am to 5 pm for 33 days) (Martin \& Bateson, 1995). Data obtained in each counting session were normalized according to the number of the bees surviving on each day of observation. 


\section{Haemolymph collection and analysis}

The second group of $45 \mathrm{O}$. bicornis females (15 for each experimental group) was kept under the same experimental conditions in order to collect haemolymph. Haemolymph was withdrawn from the thorax by insertion of a 1-ml glass microcapillary through the neck membrane (Hartfelder et al., 2013; Mazzei et al., 2016) and stored in Eppendorf tubes containing $100 \mathrm{ml}$ ethanol. Each sample was a pool from three bees belonging to each of the five time groups $(0,24,48,72$ and $96 \mathrm{~h}$ ) for each diet. The samples were centrifuged at $12000 \mathrm{~g}$ for $5 \mathrm{~min}$ to remove haemocytes and the supernatant was collected and stored at $2258 \mathrm{C}$ until analysis.

In order to determine whether the diets affected the nitrogenous profile of the haemolymph, amino acid analysis was performed by gradient high performance liquid chromatography (HPLC). Prior to analysis, the samples were dried in a vacuum centrifuge (Jouan RC 1010, Thermo Fisher Scientific, Waltham, Massachusetts, USA) to evaporate the alcohol and then resuspended with $50 \mathrm{ml}$ distilled water. HPLC was carried out with a LC 1 system (Waters Corp., Milford, Massachusetts, USA) using a Nova-Pack C18 column (150 34.6 mm, Waters Corp.) maintained at $378 \mathrm{C}$ and a 470 scanning fluorescence detector (excitation $295 \mathrm{~nm}$, detection $350 \mathrm{~nm}$, Waters Corp.). A solvent composed of triethanolamine-phosphate buffer $(\mathrm{pH} 5.0)$ in gradient with a $6: 4$ acetonitrile : water solution was used as the mobile phase at a flow rate of $1.0 \mathrm{ml} / \mathrm{min}$. The selected volume of each reconstituted sample $(10 \mathrm{ml})$ was amino acid derivatized (Cohen \& Michaud, 1993) with 6-aminoquinolyl-N-hydroxysuccinimidyl carbamate (AQC) fluorescent reagent and $0.02 \mathrm{M}$ borate buffer $(\mathrm{pH} 8.6)$ according to the AccQtag protocol (Waters Corp.). In addition to all 20 protein amino acids (with the exception of tryptophan, which is not detectable by this method), standards of eight NPAAs ( $b$-alanine, citrulline, $a$ aminobutyric acid, $b$-aminobutyric acid, GABA, hydroxyproline, ornithine and taurine) were also used. The concentration of each amino acid was calculated by comparing the area under the chromatogram peaks with standards by means of CLARITY software (DataApex, Prague, The Czech Republic).

The total amino acid concentration of each resuspended sample was calculated by summing the concentration of all the amino acids detected in that sample. As haemolymph sample volume was difficult to quantify, amino acids are reported as relative percentages of the total amino acid content of the resuspended samples.

\section{Statistical analysis}

The Shapiro-Wilk test was used to assess normality of data distribution. As the data were not normally distributed, nonparametric tests were used. Differences in survival data between the diet groups were analysed by the Wilcoxon rank test using the product-limit (Kaplan-Meier) method. Bee behavioural data were analysed by the Kruskal-Wallis $\mathrm{H}$-test followed by post hoc Mann-Whitney U-test pairwise comparisons with Bonferroni correction to assess differences between diet groups. All processing was carried out using JMP Statistics and Graphics Guide (SAS Institute, 2008) with $a$-error set at 0.05 .

\section{Acknowledgements}

Research was supported by Fondo di Finanziamento per le Attivita Base di Ricerca (FFABR) 2017 (Italian Ministry for Education, University and Research, A.F.) and Piano di Sostegno alla Ricerca (PSR) 2017 (University of Siena, M.N.).

\section{References}

Auclair, J.L. (1959) The influence of dietary amino acids on the blood amino acids of the German cockroach, Blattella germanica (L.). J Insect Physiol 3: 127-131.

Ben-Ari, Y., Khalilov, I., Kahle, K.T. and Cherubini, E. (2012) The GABA excitatory/inhibitory shift in brain maturation and neurological disorders. Neuroscientist 18: 467-486.

Bertazzini, M., Medrzycki, P., Bortolotti, L., Maistrello, L. and Forlani, G. (2010) Amino acid content and nectar choice by forager honeybees (Apis mellifera L.). Amino Acids 39: 315-318.

Bown, A.W., MacGregor, K.B. and Shelp, B.J. (2006) Gammaaminobutyrate: defence against invertebrate pests? Trends Plant Sci 11 : 424-427.

Breer, H. and Heilgenberg, H. (1985) Neurochemistry of GABAergic activities in the central nervous system of Locusta migratoria. J Comp Physiol A 157: 343-354.

Carter, C., Sharoni, S., Yehonatan, L., Palmer, R.G. and Thornburg, R. (2006) A novel role for proline in plant floral nectars. Naturwissenschaften 93: 72-79.

Cohen, S.A. and Michaud, D.P. (1993) Synthesis of a fluorescent derivatizing reagent, 6-aminoquinolyl-N-hydroxysuccinimidyl carbamate, and its application for the analysis of hydrolysate amino acids via high performance liquid chromatography. Anal Biochem 211 : 279-287.

Crailsheim, K. and Leonhard, B. (1997) Amino acids in honeybee worker haemolymph. Amino Acids 13: 141-153.

Felicioli, A. (2000) Le osmie. In Api e Impollinazione (Pinzauti M. ed.), pp 159-188. Regione Toscana, Firenze, Italy.

Felicioli, A., Kristjánsson, K., Lepore, A. and Pinzauti, M. (2002) The development of the oocyte in the cocoon imago of Osmia cornuta Latr. (Hymenoptera, Megachilidae): dependence on timing and length of cold treatment. Boll. Lab. Entomol. Agraria Filippo Silvestri 58: 21-29.

Felicioli, A., Ambroselli, S., Cilia, G. and Sagona, S. (2017) Parasitization of a wild and reared population of the solitary bee Osmia cornuta Latr. by the parasitoid Anthrax anthrax Schrank (Diptera, Bombyliidae): comparison between two types of artificial nest. J Apic Res 56: 598-605.

Gauthier, M. and Grenewald, B. (2011) Neurotransmitter systems in the honey bee brain: functions in learning and memory. In Honeybee Neurobiology and Behavior (Galizia, C., Eisenhardt, D. and Giurfa M., eds), pp. 155-169. Springer, Dordrecht, Netherlands.

Gillot, C. (2005) Entomology, 3rd edn. Springer, Dordrecht, Netherlands.

González-Teuber, M. and Heil, M. (2009) The role of extrafloral nectar amino acids for the preferences of facultative and obligate ant mutualists. J Chem Ecol 35: 459-468.

de Groot, A.P. (1953) Protein and amino acid requirements of the honey bee (Apis mellifica L.). Physiol Comp Oecol 8: 192-285.

Hartfelder, K., Bitondi, M.M.G., Brent, C.S., Guidugli-Lazzarini, K.R., Simões, Z.L.P., Stabentheiner, A. et al. (2013) Standard 
methods for physiology and biochemistry research in Apis mellifera. J Apic Res 52: 1-48.

Haydak, M.H. (1970) Honey bee nutrition. Ann Rev Entomol 15: 143-156.

Heil, M. (2011) Nectar: generation, regulation and ecological functions. Trends Plant Sci 16: 191-200.

Hrassnigg, N., Leonhard, B. and Crailsheim, K. (2003) Free amino acids in the haemolymph of honey bee queens (Apis mellifera L.). Amino Acids 24: 205-212.

Kaczorowski, R.L., Gardener, M.C. and Holtsfor, T.P. (2005) Nectar traits in Nicotiana section Alatae (Solanaceae) in relation to floral traits, pollinators, and mating system. Am J Bot 92: 1270-1283.

Krunić, N., Stanisavljević, L., Pinzauti, M. and Felicioli, A. (2005) The accompanying fauna of Osmia cornuta and Osmia rufa and effective measures of protection. Bull Insectology 58: 141-152.

Lu, Y.-X., Zhang, Q. and Xu, W.-H. (2014) Global metabolomic analyses of the hemolymph and brain during the initiation, maintenance, and termination of pupal diapause in the cotton bollworm, Helicoverpa armigera. PLoS ONE 9: e99948.

Martin, P. and Bateson, P. (1995) Measuring Behaviour: An Introductory Guide. Cambridge University Press, Cambridge, UK.

Mazzei, M., Fronte, B., Sagona, S., Carrozza, M.L., Forzan, M., Pizzurro, F. et al. (2016) Effect of 1, 3-1, 6 b-Glucan on natural and experimental deformed wing virus infection in newly emerged honeybees (Apis mellifera ligustica). PLoS ONE 11: e0166297.

Mevi-Schttz, J. and Erhardt, A. (2005) Amino acids in nectar enhance butterfly fecundity: a long-awaited link. Am Nat 165: 411-419.

Micheu, S., Crailsheim, K. and Leonhard, B. (2000) Importance of proline and other amino acids during honeybee flight (Apis mellifera carnica Pollmann). Amino Acids 18: 157-175.

Milani, N. (1988) Proprietàisiche e composizione chimica dell'emolinfa in Apis mellifera L.: una rassegna bibliografica. Apicoltura 4: 1-76.

Mitchell, B.K. and Harrison, G.D. (1984) Characterization of galeal chemosensilla in the adult Colorado beetle, Leptinotarsa decemlineata. Physiol Entomol 9: 49-56.

Nepi, M. (2014) Beyond nectar sweetness: the hidden ecological role of non-protein amino acids in nectar. J Ecol 102: 108-115.

Nepi, M., Soligo, C., Nocentini, D., Abate, M., Guarnieri, M., Cai, G. et al. (2012) Amino acids and protein profile in floral nectar: much more than a simple reward. Flora 207: 475-481.
Nicolson, S. W. (2007) Nectar consumers. In Nectaries and nectar (Nicolson, S.W., Nepi, M. and Pacini, E., eds), pp. 289342. Springer, Dordrecht, Netherlands.

Nicolson, S.W. and Thorburg, R.W. (2007) Nectar chemistry. In Nectaries and nectar (Nicolson, S.W., Nepi, M. and Pacini, E., eds), pp. 215-264. Springer, Dordrecht, Netherlands.

Nocentini, D., Pacini, E., Guarnieri, M. and Nepi, M. (2012) Flower morphology, nectar traits and pollinators of Cerinthe major (Boraginaceae-Lithospermeae). Flora 207: 186-196.

Olsen, R.W. and DeLorey, T.M. (1999) GABA synthesis, uptake and release. In Basic Neurochemistry: Molecular, Cellular and Medical Aspects, 6th edn. (Siegel, G.J., Agranoff, B.W., Albers R.W. and Uhler, M.D., eds), pp. 336. Lippincott-Raven, Philadelphia, PA.

Paoli, P.P., Donley, D., Stabler, D., Saseendranath, A., Nicolson, S.W., Simpson, S.J. et al. (2014) Nutritional balance of essential amino acids and carbohydrates of the adult worker honeybee depends on age. Amino Acids 46: 1449-1458.

Raw, A. (1972) The biology of the solitary bee Osmia rufa (L.) (Megachilidae). Ecol Entomol 124: 213-229.

Rossi, M., Fisogni, A., Nepi, M., Quaranta, M. and Galloni, M. (2014) Bouncy versus idles: on the different role of pollinators in the generalist Gentiana lutea L. Flora 209: 164-171.

SAS Institute. (2008) JMP Statistics and Graphics Guide. SAS Institute Inc., Cary, NC.

Schnee, M.E., Rauh, J.J., Buckingham, S.D. and Sattelle, D.B. (1997) Pharmacology of skeletal muscle gaba-gated chloride channels in the cockroach Periplaneta Americana. J Exp Biol 200: 2947-2955.

Shoonhoven, L.M., van Loon, J.J.A. and Dicke, M. (2005). InsectPlant Biology. Oxford University Press, Oxford.

Tasei, J.-N. and Picart, M. (1973) Observations sur le developpement d'Osmia cornuta Latr. et Osmia rufa L. (Hymenoptera Megachilidae). Apidologie 4: 295-315.

Vrzal, E.M., Allan, S.A. and Hahn, D.A. (2010) Amino acids in nectar enhance longevity of female Culex quinquefasciatus mosquitoes. J Insect Physiol 56: 1659-1664.

Wyatt, L.R. (2016) Brain homeostasis and addiction. In Homeostatic Control of Brain Function (Boyson, D. and Masino, S.A., eds), pp. 509-534. Oxford University Press, Oxford.

Yin, X.-W., Iovinella, I., Marangoni, R., Cattonaro, F., Flamini, G., Sagona, S. et al. (2013) Odorant-binding proteins and olfactory coding in the solitary bee Osmia cornuta. Cell Mol Life Sci 70: 3029-3039. 